\title{
Hierarquia social e atribuição causal em auto e hetero-avaliações de desculpas
}

\section{Social hierarchy and causal attribution in self and other- evaluation of excuses}

\section{Jerarquía social y atribución causal en auto y heteroevaluaciones de disculpas}

\author{
Raissa Damasceno* \\ Universidade de Brasília - UnB, Brasília, Distrito Federal, Brasil \\ Víthor Rosa Franco** \\ Universidade de Brasília - UnB, Brasília, Distrito Federal, Brasil
}

\section{Mauricio Miranda Sarmet***}

Instituto Federal de Educação, Ciência e Tecnologia da Paraíba - IFPB, Itabaiana, Pernambuco, Brasil

\section{Fabio I glesias $* * * *$}

Universidade de Brasília - UnB, Brasília, Distrito Federal, Brasil

\begin{abstract}
RESUMO
Em contextos sociais, quando ocorre uma falha, frequentemente usam-se as mais diversas justificativas. A questão que emerge desse contexto é quão desculpável a justificativa se apresenta a depender de sua causa (externa, interna controlável ou interna incontrolável) e da hierarquia social de quem a pede. Esta pesquisa investigou efeitos de pedidos de desculpas, feitos por pessoas de hierarquias diferentes, entre três diferentes alvos de avaliação auto (eu) e hetero (meus amigos e outros). Trezentos participantes responderam a cenários em que alguém (ora um professor, ora um aluno) se atrasa para um compromisso e oferece três tipos de desculpas que deveriam ser respondidas em três alvos de avaliação. Os resultados corroboraram o modelo de Weiner (2006), mostrando que desculpas com causas externas foram mais aceitáveis que as internas incontroláveis, seguidas das internas controláveis. Pedidos de desculpas feitos pelo professor, no entanto, foram menos aceitos. Na comparação entre auto e hetero-avaliação, confirmou-se o viés de falsa-unicidade, já que os respondentes julgaram aceitar mais os pedidos feitos a eles do que as outras pessoas aceitariam. Discutem-se aplicações para o contexto escolar, em especial para o gerenciamento de impressão em situações de hierarquia definida.
\end{abstract}

Palavras-chave: Desculpas, causas, hierarquia, julgamentos, falsa unicidade. 
Raissa Damasceno, Víthor Rosa Franco,

Mauricio Miranda Sarmet, Fabio Iglesias

\begin{abstract}
In social contexts, when a fail occurs, people usually use different justifications. This raises a question that concerns how much acceptable is this excuse, depending on its cause (external, internal controllable or internal uncontrollable) and the social status of those who use it. The present research investigated the effects of excuses given by people from different hierarchy levels considering three different targets - self and others (friends and other people). Three hundred participants judged how acceptable three excuses were in scenarios in which a person (either a teacher or a student) is coming late to an appointment. Results supported Weiner's attributional model (2006), by showing that excuses with external causes were regarded as more acceptable than those with uncontrollable internal causes, followed by controllable internal ones. Also, excuses given by professors were less accepted. When comparing different evaluations, a false uniqueness bias was confirmed, as participants judged themselves as more benevolent than others. Applications for educational context are discussed, especially for impression management in clearly defined hierarchical settings.
\end{abstract}

Keywords: Excuse, cause, hierarchy, judgments, false uniqueness.

\title{
RESUMEN
}

En contextos sociales, cuando se comete una falla, las personas empleán diferentes justificaciones. Esto plantea una pregunta que se refiere a quan aceptable es la excusa, dependiendo de su causa (externa, interna incontrolable o interna controlable) y el tipo de situación social. En el presente estudio se investigó los efectos de las excusas dadas por personas de diferentes niveles de jerarquía, considerando tres diferentes actores sociales: la persona, sus amigos y las otras personas en general). Trescientos participantes juzgaron cuanto aceptables tres excusas estaban en escenarios en los que una persona (un profesor o un estudiante) está llegando tarde a una cita. Los resultados apoyaron lo modelo atribucional de Weiner (2006), mostrando que las excusas con causas externas fueron consideradas como más aceptables que las que tienen causas internas incontrolables, seguidas de las internas controlables. Además, excusas dadas por los profesores fueran menos aceptadas. Al comparar las evaluaciones acerca de los diferentes actores sociales, se confirmó el efecto de falsa singularidad, ya que los participantes juzgaban a sí mismos como más benevolentes que los otros. Se discuten los problemas de contexto educativo, especialmente para la gestión de las impresiones en contextos jerárquicos con relaciones claramente definidas.

Palabras clave: Disculpas, causas, jerarquia, juicios, falsa singularidad.

\section{I ntrodução}

Os processos de atribuição de causalidade ocupam um papel fundamental em situações interpessoais, e podem determinar a maneira como reagimos ao observar os outros, rompendo ou seguindo normas de conduta. Desculpas são estratégias utilizadas justamente quando se comete uma falha social e pretende-se gerenciar nos outros a interpretação das causas envolvidas (Fraser, 2000; Tyler \& Feldman, 2007). Elas podem ser comunicadas como 
mais internas ou mais externas, em função da conveniência de cada situação, conferindo à atribuição um papel central nessa dinâmica (Mehlman \& Snyder, 1985; Topalli, Higgins, \& Copes, 2014). De fato, em diversos idiomas de origem latina os termos usados para pedir desculpas revelam uma tentativa de expressar que os motivos para alguma falha social estão em fatores externos ao indivíduo (ex causa) ou pelo menos que não são intencionais. Assim é com os verbos to excuse, escusare e escuser, em inglês, italiano e francês, respectivamente. Já em espanhol e em português usa-se popularmente o termo desculpa, que revela uma intenção ainda mais explícita de tentar livrar-se da culpa experienciada por quem a pede. Desculpas variam em função do contexto, da cultura, do número de pessoas envolvidas e, obviamente, da gravidade das consequências (Snyder, Higgins, \& Stucky, 2005).

O uso e a aceitabilidade de desculpas, entretanto, são também sensíveis aos julgamentos que as pessoas fazem sobre as atitudes e características dos outros, como em vários processos de cognição social e de percepção social (Fiske \& Taylor, 2013). Com base no modelo atribucional de Weiner (2006), o objetivo desta pesquisa foi testar essas diferenças com três tipos de pedidos de desculpas (causa externa, causa interna controlável e causa interna incontrolável) em diferentes níveis de hierarquia. Além de investigar a dinâmica dessa auto-avaliação, entretanto, a pesquisa enfatizou um aspecto escassamente pesquisado nas pesquisas sobre desculpas: como as pessoas avaliam a aceitabilidade de pedidos de desculpas feitos a terceiros?

Destaca-se assim o chamado viés da falsa unicidade (Suls \& Wan, 1987), no qual o indivíduo crê apresentar comportamentos e atitudes diferentes ao das outras pessoas ou grupos. Esse fenômeno foi testado dada a sua relevância em julgamentos de hetero-avaliação (Park, 2012). Como exemplo, Stern, West e Schmitt (2013) identificaram que tal fenômeno pode depender, inclusive, do conteúdo o qual se avalia, ao encontrarem que liberais subestimam a sua similaridade com outros liberais, enquanto os conservadores superestimam sua semelhança com outros conservadores. Ao perguntar o quão aceitável um pedido de desculpa é para outras pessoas (hetero-percepção) tem-se a oportunidade de verificar se 0 fenômeno também ocorre quanto a aceitabilidade de pedidos de desculpas.

As pessoas frequentemente usam crenças, atitudes $e$ comportamentos de outras pessoas como padrão de comparação e avaliação de seus próprios comportamentos e atitudes (Cialdini \& Griskevicius, 2010). Diante de situações novas ou ambíguas é esperado que ocorram processos de inferência conforme as expectativas do observador. De acordo com a teoria atribucional inicialmente desenvolvida por Heider (1958), as causas atribuídas ao 
comportamento podem ser internas (portanto inerentes a quem o emitiu) ou externas (indicando influências ambientais). Durante décadas os estudos da área se ocuparam em investigar os preditores do tipo de atribuição. No entanto, o modelo de Weiner (1986) inverteu esse foco, ocupando-se das consequências do tipo de atribuição, que passou a controlar em seus estudos. Assim, o modelo se interessa pela atribuição interna ou externa como uma variável independente, que por sua vez gera reações positivas ou negativas em que as observa, sejam elas afetivas, cognitivas ou comportamentais.

Em diversos contextos que incluem comportamento prossocial, relações de consumo, apreciação de méritos e emissão de vereditos por jurados, a teoria de Weiner (2006) e suas aplicações mostram que as pessoas julgam as ações das outras com base em três dimensões: o lócus de controle (interno ou externo), a controlabilidade (controlável ou incontrolável) e a estabilidade (estável ou instável no tempo). No caso dos pedidos de desculpas, Weiner, Amirkhan, Folkes e Verette (1987) identificaram numa série de experimentos que desculpas com causas externas (oriundas de fatores ambientais e de terceiros como, por exemplo, um engarrafamento que impede alguém de comparecer a um compromisso) são julgadas como mais aceitáveis do que desculpas de causas internas incontroláveis (por exemplo, a ausência em função de um problema de saúde). Estas últimas, por sua vez, são julgadas como mais aceitáveis do que desculpas de causas internas controláveis (ou seja, causadas pelo indivíduo e que poderiam ter sido evitadas por ele. Por exemplo, o não comparecimento a um compromisso por ter se distraído com uma atividade de lazer). Os autores também mostraram como essas estratégias são utilizadas para minimizar a raiva causada por quem é vítima de falhas em interações sociais.

Apesar da relevância da teoria e de algumas outras hipóteses dela derivadas, como mostraram Malle, Guglielmo e Monroe (2014) e Shaw, Wild e Colquitt (2003), um exame da literatura revela que a temática de desculpas ainda parece receber pouca pesquisa sistemática em psicologia social, que geralmente investiga mais as tentativas de se negar intencionalidade. Uma revisão nas bases Scielo e PePSIC, o tema é praticamente inexistente na literatura psicológica brasileira. Num dos poucos trabalhos empíricos sobre desculpas no contexto brasileiro, Saraiva e I glesias (2013) verificaram as mesmas tendências encontradas por Weiner et al. (1987), especialmente quando se considera o pedido de desculpas como uma estratégia de gerenciamento da impressão e o quão plausíveis as desculpas parecem ser para quem as recebe.

Dada a complexidade das interações pessoais, não são apenas as características das desculpas que definem sua aceitabilidade. Estudos 
de taxonomias de poder mostram que o julgamento sobre outras pessoas é claramente influenciado pelo poder social percebido nas interações. French e Raven (1959) mostraram que há bases de poder mais "leves" (p.ex., um médico que mostrar poder pela expertise em sua área ou uma mãe que oferecer recompensa ao filho) e bases mais "duras" (p.ex., um chefe que mostra poder por coerção ou um policial que tem sua função legitimada pelo Estado). Elas especificam maneiras pelas quais fatores físicos, econômicos ou sociais servem como mecanismos de controle social (Pierro, Raven, Amato, \& Bélanger, 2013). Depreende-se, portanto, que em relações hierárquicas, os pedidos de desculpas podem ser formulados e recebidos de formas distintas.

Uma relação que pode ser considerada tipicamente hierárquica e que envolve diferenças de poder é a dinâmica professor-aluno (Martinelli $\&$ Schiavoni, 2009). Dillon (1998) mostrou que a aceitabilidade de desculpas, por exemplo, por ter faltado a uma aula, varia se o pedido é feito por um aluno ou por um professor, evidenciando diferenças entre o que é esperado de seus papéis sociais. É fundamental investigar esses fenômenos no contexto acadêmico, dada a frequência de falhas em interações sociais e a maneira como muitas vezes se desdobram em problemas maiores, como punições legítimas, sentimentos de injustiça, processos disciplinares e até agressões. De fato, dados da Organização para a Cooperação e o Desenvolvimento Econômico listam o Brasil, entre 40 países, como aquele em que mais se verificam conflitos agressivos verbais ou físicos entre professores e alunos (OCED, 2014), que poderiam ser amenizados com processos de gerenciamento de conflitos em relações hierárquicas, como é o caso do uso de desculpas.

Aceitar ou não um pedido de desculpas envolve processos de julgamento social tipicamente investigados em cognição social. Muitas vezes esses fenômenos mostram uma grande assimetria entre julgamentos do ator e julgamentos do observador (Sloman \& Lagnado, 2015). Além disso, há diversos fatores que podem influenciar o quanto determinado julgamento é percebido como consensual em um determinado grupo social. Suls e Wan (1987), por exemplo, demonstraram que as pessoas que acreditam possuir atributos indesejáveis tendem a crer que seu julgamento é similar ao do restante do grupo (falso consenso), enquanto pessoas que acreditam possuir atributos desejáveis subestimam o consenso e percebem seus julgamentos como singulares (falsa unicidade). Considerando o contexto de desculpas, ser uma pessoa que expressa atitudes mais favoráveis a diferentes justificativas (mesmo aquelas consideradas pouco adequadas) denota um conjunto de atributos socialmente desejáveis. Trata-se, portanto, de uma estratégia de gerenciamento de impressão, como as identificadas há muito na microssociologia de Goffman (2009), utilizadas em contextos sociais 
que se caracterizam pela constante presença física imediata de outras pessoas.

Considerando esses elementos teórico-conceituais, esta pesquisa buscou identificar de que forma a hierarquia numa interação interpessoal influencia a aceitabilidade de pedidos de desculpas e a ocorrência do viés de falsa unicidade. Três hipóteses principais foram delineadas. Primeiramente, dado o corpo de evidências levantadas por Weiner (2006), espera-se que desculpas externas sejam tidas como mais aceitáveis do que desculpas internas e que desculpas internas incontroláveis sejam tidas como mais aceitáveis do que desculpas internas controláveis. Em segundo lugar, dado que a aceitabilidade de um pedido de desculpas é sensível ao fato de quem a oferece ser um aluno ou um professor, espera-se que o pedido do professor tenha menor aceitabilidade que 0 do aluno. Por fim, considerando que a aceitação de desculpas pode ser entendida como uma estratégia de gerenciamento de impressão, espera-se que os participantes se julguem mais benevolentes do que as outras pessoas.

\section{Método}

\subsection{Participantes}

Participaram do estudo 300 estudantes universitários (57,7\% homens), de diferentes cursos, recrutados de maneira não-randômica em um campus universitário. O tamanho amostral foi baseado nas pesquisas revisadas por Weiner (2006). Os participantes relataram uma média de 23,78 anos de idade $(D P=7,78)$. Um participante foi descartado da amostra, por ter sido o único a não responder a todas as questões dos instrumentos.

\subsection{I nstrumentos e procedimentos}

$\mathrm{Na}$ forma de um delineamento entre-sujeitos foram utilizados dois tipos de questionários que apresentavam cenários distintos, mas envolvendo uma mesma situação de compromisso de reunião. Metade da amostra recebeu um cenário em que uma autoridade (professor) pede desculpas por ter chegado atrasado; a segunda metade recebeu um cenário em que uma não-autoridade (aluno) pede desculpas por ter chegado atrasado. Três tipos de desculpas foram manipuladas nos cenários, com base em Weiner (1986). A primeira era uma desculpa de causa externa ao indivíduo ("Houve um acidente de trânsito e peguei um engarrafamento"), enquanto as outras duas eram de causa interna, sendo uma incontrolável ("Estou doente e acabei passando mal, por isso me atrasei") e a outra 
controlável ("Me diverti até tarde da noite, dormi pouco e perdi a hora."). Com o intuito de evitar um possível efeito de ordenamento, foram utilizadas diferentes versões do instrumento, nas quais se alternou a ordem de apresentação de cada tipo de desculpa, resultando em seis versões diferentes de cada instrumento.

O participante respondia o quão aceitável julgava cada uma das três opções de pedido de desculpas, em ambos os questionários, de acordo com uma escala bipolar de cinco categorias ("totalmente inaceitável" a "totalmente aceitável") e para diferentes alvos: o próprio participante (variável de autopercepção), um amigo e um desconhecido (variáveis de heteropercepção).

Foram cumpridos os critérios éticos para pesquisas com seres humanos, com base no projeto aprovado pelo comitê de ética em pesquisa da instituição dos autores, assegurando uma participação anônima, voluntária e ausente de qualquer risco ao participante. Somente fizeram parte da amostra os participantes que forneceram consentimento em participar da pesquisa, após declararem que estavam esclarecidos e de acordo com o desenvolvimento da pesquisa. Além disso, os participantes foram informados que poderiam desistir a qualquer momento.

\subsection{Plano de análise dos dados}

Nos procedimentos de análise exploratória dos dados constatou-se a falta de normalidade na distribuição das variáveis medidas. Portanto, foram utilizadas as correspondentes não-paramétricas do teste $t$ de amostras independentes e de amostras dependentes (Mann-Whitney e signed rank de Wilcoxon, respectivamente) e da ANOVA para medidas repetidas (ANOVA de Friedman). Inicialmente verificou-se a possibilidade de um efeito do ordenamento na apresentação dos tipos de desculpa, mas as tendências reveladas para cada subamostra apresentaram-se iguais. Decidiu-se então por utilizar a amostra completa para o restante das análises, independente da ordem em que as desculpas foram apresentadas.

\section{Resultados}

A avaliação dos participantes quanto à aceitabilidade mudou significativamente de acordo com o tipo de pedido de desculpa, quando testados via ANOVA de Friedman, $X^{2}(2)=287,51, p<0,001$. Como estratégia post hoc, testes de Wilcoxon com correção Bonferroni do nível de significância para 0,016 (Field, 2013) apontaram que o julgamento da aceitabilidade do pedido feito ao participante com uma justificativa de causa externa (ranking médio $=$ 96,66) foi maior do que para a desculpa de causa interna 
incontrolável (ranking médio $=96,08$ ), $T=13.435,50, z=-5,67, p=$ $0,001, r=0,39$, e para a desculpa de causa interna controlável ( ranking médio $=92,45$ ) $, T=2.866,00, z=-12,10, p<0,001, r=$ 0,70 . As desculpas de causa incontrolável foram mais aceitas que as desculpas de causa controlável, $T=35.775,50, z=-13,01, p<$ $0,001, r=0,75$.

Para testar o efeito da hierarquia, a comparação dos três pedidos de desculpas, feitos ora por um professor ora por um aluno, foi feita apenas usando a autoavaliação (o quanto que o participante julgou o pedido aceitável para si), com base em testes de Mann-Whitney. Quando a desculpa era de causa externa, os participantes aceitaram menos o pedido feito pelo professor (ranking médio $=141,25$ ) do que pelo aluno (ranking médio $=159,75$ ) $, U=9.862,50, z=-1,92, p=$ $0,03, r=0,11$. Na condição de causa interna incontrolável, não houve diferenças significativas no pedido de desculpa feito por um professor (ranking médio $=146,80$ ) ou por um aluno (ranking médio $=154,20), U=10.694,50, z=-0,04, p=0,21, r=0,15 . \mathrm{Na}$ condição em que o pedido de desculpa era de causa interna controlável, os participantes também julgaram que o pedido de desculpa feito por um professor (ranking médio $=138,40$ ) é menos aceitável do que se feito pelo aluno (ranking médio $=162,60$ ),$U=$ 9.434,50, $z=-2,56, p=0,005, r=0,15$.

Para testar o viés de falsa unicidade, foram comparadas as auto e heteropercepções de aceitabilidade dos três tipos de desculpa via ANOVAs de Friedman. Elas diferiram no pedido de causa externa, $X^{2}(2)=53,86, p<0,001$. No entanto, comparações múltiplas post hoc apontaram que os participantes julgaram aceitar mais (ranking médio $=84,21$ ) do que os seus amigos (ranking médio $=72,75$ ),$T=$ $2.430,50, z=-3,12, p=0,001, r=0,18$, e mais do que desconhecidos (ranking médio $=66,85$ ), $T=3.177,00, z=-6,00, p$ $<0,001, r=0,35$. Quando o pedido de desculpa era de causa interna incontrolável, $X^{2}(2)=42,41, p<0,001$, a auto (ranking médio = 93,05 ) e a heteropercepção (ranking médio $=84,80$ ) em relação aos amigos não se diferenciaram, $T=4.392,50, z=-0,49, p=n s, r=$ 0,03 . Quando comparado a um desconhecido (ranking médio $=$ $66,95)$, entretanto, os participantes julgaram que o pedido era mais aceitável para si mesmos, $T=4.125,50, z=-4,68, p<0,001, r=$ 0,27 . No pedido de desculpa de causa interna controlável, a aceitabilidade do pedido também mudou significativamente, $x^{2}(2)=$ $64,97, p<0,001$. Os participantes julgaram que o pedido seria mais aceitável para eles (ranking médio $=85,95$ ) do que para um amigo ( ranking médio $=68,37$ ) $, T=1.955,00, z=-4,33, p<0,001, r=$ 0,25, e para um desconhecido (ranking médio $=51,56), T=$ 3.816,00, $z=-6,34, p<0,001, r=0,37$. 


\section{Discussão}

Este estudo investigou se a hierarquia numa interação social influencia a aceitabilidade de diferentes tipos de pedidos de desculpas, assim como a ocorrência do viés de falsa unicidade nessas avaliações. De acordo com o previsto por Weiner (2006), e em consonância com o estabelecido pela primeira hipótese, observaramse diferenças substanciais na causalidade que cada pedido representava. Levando-se em conta que as desculpas podem ser entendidas como estratégias sociais no gerenciamento de impressão para falhas ou comportamentos inadequados (Weiner et al., 1987), desculpas cuja causa foge do controle do indivíduo que se desculpa tenderam a ser interpretadas como mais aceitáveis do que aquelas sobre as quais ele possui maior gestão. Isso explica porque a desculpa de causa externa foi considerada a mais aceitável, seguida pela de causa interna incontrolável e por último a de causa interna controlável, corroborando as evidências de estudos anteriores realizados no Brasil (Saraiva \& I glesias, 2013).

Ao analisar o efeito da hierarquia na aceitabilidade das desculpas, os resultados evidenciaram que o mesmo pedido feito pelo professor foi menos aceito do que quando feito pelo aluno, o que corroborou a segunda hipótese do estudo. Para compreender tal relação, é importante levar em conta as evidências de que o papel hierárquico que o indivíduo ocupa em um contexto social traz consigo expectativas com relação às suas atribuições, comportamentos e, principalmente, quanto poder o indivíduo possui para gerir este contexto (Raven, Schwarzwald, \& Koslowsky, 1998). Considerando que a relação entre professores e alunos é caracterizada pela diferença hierárquica (Martinelli \& Schiavoni, 2009) e que há uma diferença entre expectativas para cada um destes papéis (Dillon, 1998), é razoável concluir que os participantes tenham sido mais rigorosos ao aceitar desculpas controláveis pelo indivíduo que possui maior poder. Ainda que desculpas com causas externas sejam geralmente tomadas como mais aceitáveis, elas também podem ser entendidas como falsas justificativas para comportamentos inadequados de indivíduos em posições hierárquicas mais altas (pois supostamente têm maior poder para influenciar eventos externos), resultando em um maior rigor por parte de quem os julga (Lee \& Tiedens, 2001).

Com relação à última hipótese, os resultados apontaram diferenças lineares nos alvos de avaliação para o viés da falsa unicidade nas desculpas externas e internas controláveis, revelando maiores escores na auto ("eu") do que hetero-avaliações ("amigos" e "desconhecidos") de aceitabilidade. No caso das internas incontroláveis, os três alvos não se mostraram todos diferentes entre si, mas permaneceu a tendência de maior auto do que hetero- 
avaliações. Isso confirma a direção hipotetizada por Suls e Wan (1987) para as situações em que o respondente poderia ser mais benevolente, ou especial, do que amigos ou desconhecidos. Embora alguns estudos recentes tenham retomado o interesse pelo viés de falsa unicidade (p.ex., Kulig, 2013), o fenômeno ainda é pouco investigado e merece uma agenda de pesquisa que possa ser articulada com processos de gerenciamento de impressão, como no caso das desculpas.

Apesar dos resultados apresentados neste estudo, é preciso reconhecer alguns elementos que poderiam aprimorar a compreensão do fenômeno de aceitabilidade de desculpas. Em especial, entende-se que traços de personalidade e outras características pessoais forneceriam dados valiosos de interpretação dos resultados. Variáveis como empatia, agradabilidade, evitação de conflitos, desejabilidade social e comportamentos de ajuda, entre outras, têm sido apontadas como fundamentais para explicar comportamentos sociais (Leary \& Hoyle, 2009). Além disso, a congruência entre as características da amostra utilizada neste estudo e um dos papéis hierárquicos (estudantes universitários) pode ter gerado um efeito de favorecimento do endogrupo, à semelhança do que se observa nas pesquisas sobre comportamento grupais (Abrams \& Hogg, 2006).

Destacando especificamente as situações do contexto escolar e acadêmico, Weiner (2003) caracterizou a sala de aula como um tribunal, em que os atores envolvidos estão constantemente fazendo julgamentos sobre os comportamentos dos outros, sobre sistemas de recompensa e punição, assim como atribuições de responsabilidade. Embora a metáfora possa soar exagerada, ou mesmo negativa, estudos com base nessa lógica podem ajudar a gerar insumos para aprimorar as relações de alunos e professores. Desculpar-se é uma estratégia de gerenciamento de impressão usada muitas vezes de maneira banalizada (Burke \& Rau, 2007), por isso é essencial que se conheçam as motivações e os efeitos dos diferentes tipos de desculpa, para que se possam garantir melhores julgamentos e, por consequência, comportamentos interpessoais mais harmônicos.

\section{Referências}

Abrams, D., \& Hogg, M. A. (2006). Social identifications: A social psychology of intergroup relations and group processes. New York: Routledge.

Burke, L. A., \& Rau, B. (2007). Managing chronic excuse-making behaviors of faculty applying Schlenker's responsibility triangle. Educational Management Administration \& Leadership, 35(3), 415-428. 
Cialdini, R. B. \& Griskevicius, V., (2010) Social Influence. In Baumeister, R. F., \& Finkel, E. J. (Eds.). Advanced social psychology: The state of the science. New York: Oxford University Press.

Dillon, K. M. (1998). Reasons for missing class. Psychological Reports, 83, 435-441.

Field, A. (2013). Discovering statistics using IBM SPSS statistics. London: Sage.

Fiske, S. T., \& Taylor, S. E. (2013). Social cognition: From brains to culture. New York: McGraw-Hill.

Fraser, C. O. (2000). The social goals of excuses: Self serving attributions or politeness strategies. Journal of Applied Social Psychology, 30(3), 599-611.

French, J. R. P. J r, \& Raven, B. H. (1959). The bases of social power. In D. Cartwright (Ed.). Studies in social power (pp. 150-167). Ann Arbor: University of Michigan Press.

Goffman, E. (2009). Relations in public: Microstudies of the public order. New Brunswick, NJ: Transaction Publishers (publicado originalmente em 1971).

Heider F. (1958). The psychology of interpersonal relations. New York: Wiley.

Kulig, J. W. (2013). What's in a name? Our false uniqueness. British Journal of Social Psychology, 52, 173-179.

Leary, M. R., \& Hoyle, R. H. (2009). Situations, dispositions, and the study of social behavior. In Leary, M. R., \& Hoyle, R. H. (Eds.). Handbook of individual differences in social behavior. (pp. 311). New York: Guilford.

Lee, F., \& Tiedens, L. Z. (2001). Who's being served? "Self-serving" attributions in social hierarchies. Organizational Behavior and Human Decision Processes, 84(2), 254-287.

Malle, B. F., Guglielmo, S., \& Monroe, A. E. (2014). A theory of blame. Psychological Inquiry, 25, 147-186.

Martinelli, S. C., \& Schiavoni, A. (2009). Percepção do aluno sobre sua interação com o professor e status sociométrico. Estudos de Psicologia (Campinas), 26(3), 327-336.

Mehlman, R. C., \& Snyder, C. R. (1985). Excuse theory: A test of the self-protective role of attributions. Journal of Personality and Social Psychology, 49(4), 994-1001.

Organisation for Economic Cooperation and Development (2014). Education at a glance 2014: OECD indicators. Paris: OECD Publishing.

Park, H. S. (2012). Culture, need for uniqueness, and the false consensus effect. Journal of Social, Evolutionary, and Cultural Psychology, 6(1), 82-92.

Pierro, A., Raven, B. H., Amato, C., \& Bélanger, J. J. (2013). Bases of social power, leadership styles, and organizational commitment. 
International Journal of Psychology, 48(6), 1122-1134.

Raven, B. H., Schwarzwald, J., \& Koslowsky, M. (1998). Conceptualizing and measuring a power/interaction model of interpersonal influence. Journal of Applied Social Psychology, 28(4), 307-332.

Saraiva, R. B., \& Iglesias, F. (2013). Julgamentos de plausibilidade e reações emocionais a desculpas. Interação em Psicologia, $17(2), 163-170$.

Shaw, J. C., Wild, E., \& Colquitt, J. A. (2003). To justify or excuse?: A meta-analytic review of the effects of explanations. Journal of Applied Psychology, 88(3), 444.

Sloman, S. A., \& Lagnado, D. (2015). Causality in thought. Annual Review of Psychology, 66, 223-247.

Snyder, C. R., Higgins, R. L., \& Stucky, R. J. (2005). Excuses: Masquerades in search of grace. Clinton Corners, NY: Percheron.

Stern, C., West, T. V., \& Schmitt, P. G. (2013). The liberal illusion of uniqueness. Psychological Science, 25(1), 137-144.

Suls, J., \& Wan, C. K. (1987). In search of the false-uniqueness phenomenon: Fear and estimates of social consensus. Journal of Personality and Social Psychology, 52(1), 211.

Topalli, V., Higgins, G. E., \& Copes, H. (2014). A causal model of neutralization acceptance and delinquency making the case for an individual difference model. Criminal Justice and Behavior, 41(5), 553-573.

Tyler, J. M., \& Feldman, R. S. (2007). The double-edged sword of excuses: When do they help, when do they hurt? Journal of Social and Clinical Psychology, 26, 659-688.

Weiner, B. (1986). An attributional theory of motivation and emotion. New York: Springer-Verlag.

Weiner, B. (2003). The classroom as a courtroom. Social Psychology of Education, 6, 3-15.

Weiner, B. (2006). Social motivation, justice, and the moral emotions: an attributional approach. Mahwah, N.J: Erlbaum.

Weiner, B., Amirkhan, J., Folkes, V. S., \& Verette, J. A. (1987). An attributional analysis of excuse giving: Studies of a naive theory of emotion. Journal of Personality and Social Psychology, 52(2), 316.

\section{Endereço para correspondência \\ Raissa Damasceno \\ Universidade de Brasília \\ Instituto de Psicologia - Campus Universitário Darcy Ribeiro \\ ICC Sul A1-116, Laboratório de Psicologia Social, CEP 70910-900, Brasília - DF, Brasil \\ Endereço eletrônico: damasceno.rc@gmail.com}

Víthor Rosa Franco 
Raissa Damasceno, Víthor Rosa Franco,

Universidade de Brasília

Instituto de Psicologia - Campus Universitário Darcy Ribeiro

ICC Sul A1-116, Laboratório de Psicologia Social, CEP 70910-900, Brasília - DF, Brasil

Endereço eletrônico: vithorfranco@gmail.com

\section{Mauricio Miranda Sarmet}

Instituto Federal de Educação, Ciência e Tecnologia da Paraíba

Rua Vereador Martins de Carvalho, s/n, Centro, CEP 58360-000, Itabaiana - PB, Brasil

Endereço eletrônico: mauricio.sarmet@ifpb.edu.br

\section{Fabio I glesias}

Universidade de Brasília

Instituto de Psicologia - Campus Universitário Darcy Ribeiro

ICC Sul A1-116, Laboratório de Psicologia Social, CEP 70910-900, Brasília - DF, Brasil

Endereço eletrônico: iglesias@unb.br

Recebido em: 07/10/2015

Reformulado em: 19/05/2016

Aceito para publicação em: 23/05/2016

\section{Notas}

* Aluna de mestrado do Programa de Pós-Graduação de Psicologia Social, do Trabalho e das Organizações da Universidade de Brasília (PSTO - UnB). Graduada em Psicologia pela Universidade de Brasília - Brasília (2014). Pesquisadora do Núcleo de Estudos e Pesquisas em Inovação e Estratégia da Universidade de Brasília (NINE - UnB).

** Aluno de mestrado do Programa de Pós-Graduação de Psicologia Social, do Trabalho e das Organizações da Universidade de Brasília (PSTO - UnB). Graduado em Psicologia pela Universidade de Brasília - Brasília (2015). Pesquisador do Núcleo de Estudos e Pesquisas em Inovação e Estratégia da Universidade de Brasília (NINE - UnB).

*** Professor do Instituto Federal de Educação, Ciência e Tecnologia da Paraíba. Graduado em Psicologia pela Universidade de Brasília (2000) e mestre em Psicologia Social, do Trabalho e das Organizações pela Universidade de Brasília (2003). Aluno de doutorado do Programa de Pós-Graduação de Psicologia Social, do Trabalho e das Organizações da Universidade de Brasília (PSTO - UnB).

**** Professor do Programa de Pós-Graduação em Psicologia Social, do Trabalho e das Organizações da Universidade de Brasília (PSTO - UnB). Chefe do Departamento de Psicologia Social e do Trabalho do Instituto de Psicologia da Universidade de Brasília (PST - IP - UNB). 